\title{
De la experiencia digital a la necesidad formativa
}

\author{
Alain Massieu Paulin
}

\section{Resumen}

Vivimos tiempos complicados donde distintas formas de expresión se manifiestan en las redes sociales, propiciados por el uso del internet. Uno de estos sitios es YouTube, plataforma en la que los usuarios se convierten en generadores de contenido. De igual forma, este espacio ha significado, para algunos, una fuente de importantes ingresos económicos, lo cual ha resultado en la creación del concepto de youtuber; y para otros, una oportunidad para continuar sus estudios.

Este texto relata los motivos por los que yo, Alain Massieu Paulin, médico general y actual profesor en la Facultad de Medicina de la Universidad Nacional Autónoma de México (UNAM), decidí ingresar a la Maestría en Educación en Ciencias de la Salud. Igualmente describe mi experiencia profesional como docente y menciona lo que he aprendido durante el trayecto, como las características de la generación millennial, el uso de YouTube en educación en ciencias de la salud y las distintas teorías educativas.

Palabras clave: medicina, maestría, YouTube, youtuber, millennial, educación..

\section{From the digital experience to the need of academic training}

\begin{abstract}
Currently, these are complicated times where different forms of expression manifested in social networks, propitiated using the internet. One of these sites is YouTube, a platform in which users become content generators. Similarly, this space has meant for some, a major income source, which has resulted in the creation of the concept of youtuber, and for others, an opportunity to continue his studies.

The following text is intended to tell the reasons why Alain Massieu Paulin, general practitioner and current professor in the Faculty of Medicine of the UNAM, decided to enter the master's in education in health sciences. Also describe his professional experience as a teacher and mention what he has learned along the way, as the characteristics of the millennial generation, the use of YouTube in education and the different theories of educational sciences.
\end{abstract}

Key words: medicine, master, YouTube, youtuber, millennial, education. 


\section{Alain Massieu Paulin}

massieu@gmail.com

Médico general, profesor en la Facultad de Medicina de la Universidad Nacional Autónoma de México (UNAM). Actualmente cursa la Maestría en Educación en Ciencias de la Salud en la UNAM.

\section{Introducción}

En la actualidad se viven tiempos complejos: por un lado, cada vez hay más personas que abogan por la diversidad, los derechos universales o la libertad; por el otro, viejos horrores vuelven a salir de las sombras proyectadas por ideologías de extrema derecha, por medio de aparentes alternativas como la alt-right (derecha alternativa) asociada a un presidente que justifica la discriminación. Muchas de las expresiones de tolerancia o de odio han encontrado en internet la tecnología idónea para manifestarse, reproducirse y amplificarse. Y es justamente esta tecnología, junto con las distintas redes sociales que en ella habitan, la que ha permitido a algunos construir empresas multimillonarias; y a otros, como mi caso, continuar con los estudios de posgrado.

Soy Alain Massieu Paulin, médico general, profesor de la Facultad de Medicina de la Universidad Nacional Autónoma de México (UNAM) y actual estudiante de la Maestría en Educación en Ciencias de la Salud. En este texto pretendo narrar lo que me llevó a continuar mis estudios, lo que he aprendido en el camino $y$, tal vez con ello podré responder si soy un youtuber.

\section{Acercamiento a la docencia}

Durante mi internado médico (ese quinto año de la carrera de medicina que todo estudiante debe enfrentar) el interés por la parte clínica de la profesión fue disminuyendo, pero nuevas ideas y preguntas surgieron. Me interesaba mucho comprender la forma en que, como estudiantes, aprendíamos en ambientes tan caóticos como los hospitales o cuánto de lo que veíamos en los ciclos básicos (los dos primeros años de la carrera) se quedaba en nuestras mentes. Estas inquietudes, y el deseo de intervenir en la formación de los estudiantes, así como las venta-

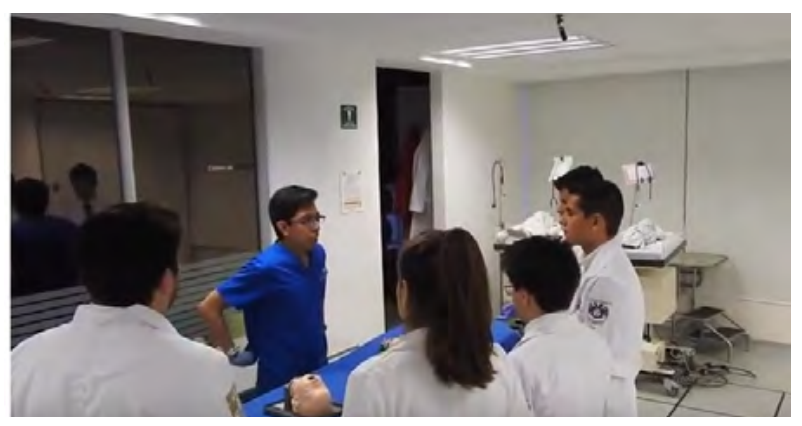
jas de permanecer en la ciudad de México (CDMX), me llevaron a elegir como plaza para realizar el servicio social el Centro de Enseñanza y Certificación de Aptitudes Médicas (CECAM) de la UNAM. En este espacio, dedicado a la formación de futuros médicos por medio de la simulación, tuve un gran acercamiento a los 
Da clic en la imagen.

Foto 1.

Exponiendo en el Coloquio de Investigación. Foto: Carolina

García Rivera estudiantes y sentí orgullo de poder ser parte de su crecimiento. También fue en ese momento que tomé la difícil decisión de no hacer una especialidad médica, de no seguir el camino que muchos médicos escogen.

Por fortuna, justo cuando terminé la carrera me ofrecieron trabajo como profesor de

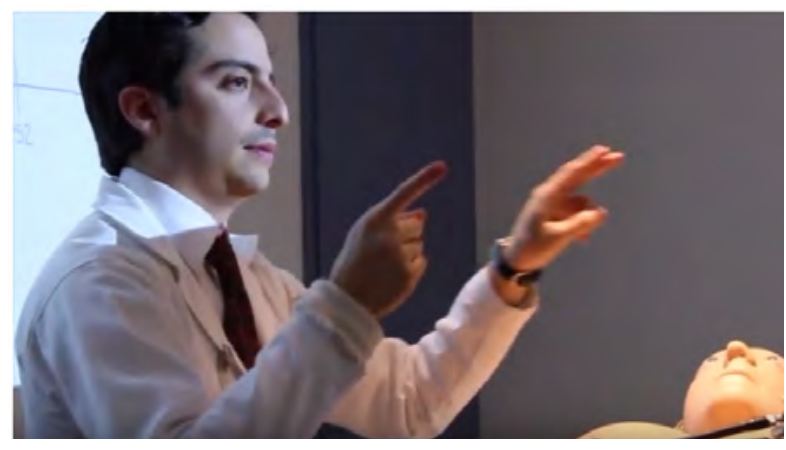
las asignaturas de Integración Básico Clínica I y II (IBC I y II) en la Facultad de Medicina (UNAM). En estas asignaturas se utiliza el modelo de Aprendizaje basado en problemas, el cual busca ser una alternativa frente a la manera magistral de dar clases que todos conocemos. Mas, laborando como profesor de estos programas, me di cuenta que nunca nos habían preparado como futuros educadores.

\section{El vídeo que lo comenzó todo}

Durante una de las prácticas en el CECAM noté que los estudiantes salían con dudas o con ganas de repetirla, pero por cuestiones de logística esto era imposible. La solución inmediata a ello: hacer un vídeo. Así que en una sesión dirigida por el Dr. Emilio Montes saqué mi iPhone 5 c y presioné el botón de grabar. Acto seguido: lo subí a mi canal de YouTube - ¿acaso no es lo que se hace hoy en día después de grabar un video?

Empecé a compartir ese video con mis estudiantes como un material adicional a la clase y la respuesta que obtuve fue sorprendente: las reproducciones del video subían, la página se llenaba de comentarios positivos e incluso en la Facultad se acercaron estudiantes para comentarme que les había servido el video. Eventualmente, la Dra. Sara Morales López se enteró de lo que había hecho y me sugirió que hiciera algunos videos adicionales para la asignatura. La res-

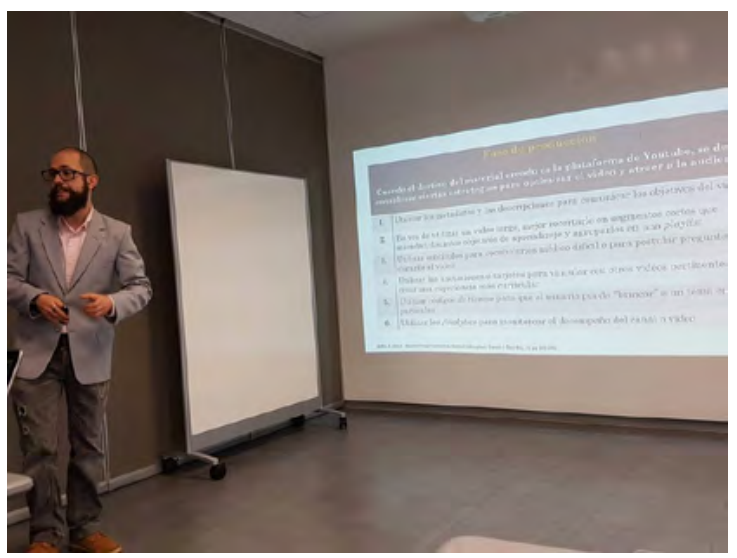

puesta que obtuve de los estudiantes fue semejante a la inicialmente descrita.

Todo esto de los videos llevó a hacerme algunas preguntas: ¿de qué forma afectaban en el aprendizaje?, ¿por qué les gustaban tanto a los estudiantes?, ¿cómo se hace realmente un video educativo? y, más importante, ¿realmente estaban funcionando? 


\section{Qué "dice" la evidencia}

Después de hacer todos los trámites, realizar entrevistas, contestar exámenes y presentar mi propuesta de proyecto... fui aceptado en el posgrado. También tuve el privilegio de que la Dra. Frida Díaz Barriga Arceo aceptará ser mi tutora y la fortuna de contar con compañeros con los cuales conversar, reír y salir después de clases.

Sin embargo, cuando uno se encuentra ya en la maestría, afirmaciones como "todo el mundo ve vídeos", "los jóvenes se la pasan en internet", etcétera, no son suficientes para contestar preguntas de investigación o pretender medir el efecto producido por un video educativo como herramienta didáctica para el desarrollo de

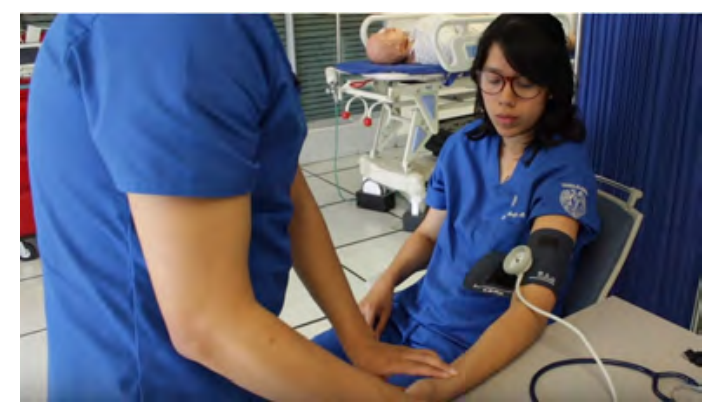
una habilidad clínica en los estudiantes de IBC II. En estos niveles, nos vemos obligados a investigar, conocer e indagar qué "dice" la evidencia científica al respecto, como siempre nos recuerda nuestro profesor el Dr. Melchor Sánchez Mendiola.

\section{Millennials, posmodernismo e internet}

Lo primero que me interesó investigar, versó sobre la actual generación de estudiantes que estamos formando los profesores (y a la que por definición pertenezco) y cómo consumen información o producen conocimiento.

Los millennials, término acuñado por los sociólogos Neil Howe y William Strauss, somos los individuos nacidos entre los años 1982 y 2000. Algunos de nuestros principales valores son la autenticidad, el estatus social (que pueda reflejarse en las redes sociales) y mantener cierto estilo de vida que constantemente se ve amenazado por las crisis financieras. También solemos ser estereotipados, por las generaciones pasadas, de flojos; pero la realidad es que tememos por nuestro futuro, en el que se vislumbra no tener casa propia ni pensión alguna.

Como estudiantes estamos motivados y somos respetuosos, seguimos las reglas establecidas, hemos sido criados en ambientes sobreprotectores, buscamos 
adquirir nuevos talentos y alcanzar metas. Trabajamos en equipo y gustamos utilizar casos y mapas mentales, preferimos obtener información por medios tecnológicos y saber para qué sirven los conocimientos adquiridos; deseamos realimentación inmediata por parte del cuerpo docente. (Elam, Stratton y Gibson, 2007; Roberts, Newman y Schwartzstein, 2012; Turner, Prihoda, English, Chismark y Jacks, 2016; Wilson y Gerber, 2008). Sin embargo, para ..he comprendido
que la Educación
es mucho más que
dar clases tomar
clases. La Educación
es un universo de
posibilidades que hay
que explorar... intentar entender a mi generación, debemos primero conocer el contexto postmoderno en el que nacimos.

En las últimas dos décadas del siglo xx hubo un cambio radical en el régimen social, económico y cultural conocido en sociología como la postmodernidad. En ésta se rompen las ideas de utopías, del progreso, se cuestionan a los grandes líderes o héroes (surgiendo infinidad de pequeños e efímeros ídolos) y a las religiones; se reconocen las individualidades y se valora la idea de pluralidad o de igualdad. Por otro lado, como lo diría el filósofo Gianni Vattimo, los medios de comunicación masiva, así como las tecnologías digitales, son un factor decisivo para conformar y entender a la sociedad postmoderna (Donovan, 2011).

Actualmente, entre las tecnologías informativas más usadas, se encuentran las distintas redes sociales como: Facebook, Twitter, Snapchat, Instagram, Tumblr y YouTube. En el caso de nuestro país, de acuerdo a la Encuesta Nacional 2015 sobre Disponibilidad y Uso de las Tecnologías de la Información en los Hogares Mexicanos (ENDUTIH) elaborada por el INEGI, existen 62.4 millones de personas (57.4\% de la población nacional) que cuentan con conexión a internet, de los que $70.7 \%$ se conectan a través de telefonía móvil; $98.2 \%$ utilizan al menos una red social; $76.6 \%$ consumen contenidos audiovisuales y únicamente $42.9 \%$ consume contenido textual. El uso de la internet es más frecuente en la población de 18 a 34 años (76.5\%), intervalo de edad de quienes son llamados generación millenial; esto contrasta con el 46.7\% de usuarios entre 35 a 59 y de 14\% de cibernautas mayores a 60 años (Villamil, 2016). Estos datos concuerdan con la encuesta de la Asociación Mexicana de Internet (AMIPCI, 2015) que además aporta el promedio de conexión a Internet por día en México: 6 horas 11 minutos (y cada año se incrementa).

\section{YouTube y medicina}

YouTube es una red social en la que las personas pueden almacenar y compartir videos. Tiene más de mil millones de usuarios y cada día, a nivel mundial, sus contenidos en vídeo se ven cientos de millones de horas y se generan miles de 
Figura 1.

Teoría cognoscitiva del aprendizaje multimedia de Richard Mayer (Latapie, 2007). millones de reproducciones. En ese mismo sentido, al día se ven cuatro mil millones de videos (100 millones desde celulares), cada minuto se suben más de 48 horas de video en 38 idiomas y en 25 países distintos (La redacción Proceso, 2012). Dicho espacio es ya una opción frente a los medios audiovisuales tradicionales como, por ejemplo, la televisora mexicana Televisa (que ha visto disminución significativa en sus ratings gracias a las tendencias de consumo millennial) y también es un espacio de reivindicación creativa, en la que el usuario se percibe como youtuber; es decir, como persona activa que busca, crea, adapta y difunde contenidos. La plataforma ha permitido hacer más eficientes sus generadores de contenido, al punto de que hoy podemos hablar de ejemplos de éxito como el de la joven mexicana Yuya, cuyo canal de belleza en marzo del 2015 produjo $\$ 41,475$ usD por las visitas alcanzadas (Ybarra, 2015).

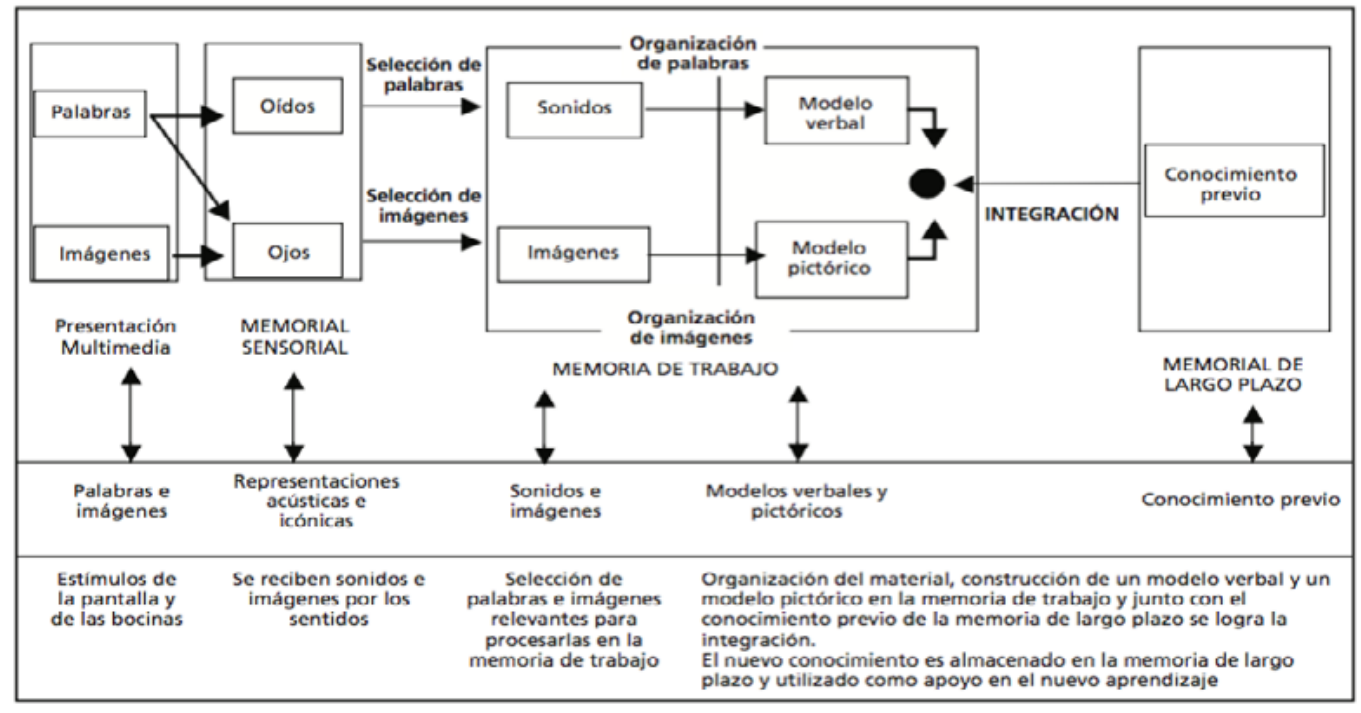

Formada a partir de la figura proporcionada por Mayer (2005, p.37) para representar la Teoria Cognoscitiva del aprendizaje multimedia y de la tabla de las cinco formas de representación (Mayer, 2005, p.42).

La plataforma no sólo sirve para ver videos de gatitos o denunciar a \#ladies y \#gentlemens. Entre las diversas propuestas creativas, y distintos tipos de contenido que se alojados en YouTube, se encuentran videos didácticos que pueden interesar al estudioso de las Ciencias de la Salud. Estas expresiones intelectuales suelen ser atractivas para el estudiante ya que son medios versátiles de presentación, que permiten combinar diversos elementos como: imágenes, textos, sonidos; además resulta ser un medio más cómodo para ilustrar ejemplos y capturar eventos reales a los que un estudiante no podría acceder fácilmente. Actualmente podemos encontrar literatura (Barry et al., 2016; Jaffar, 2013; Jaffar, 2012; Rapp et al., 2016) que menciona las ventajas de usar dicha plataforma específicamente para educación médica: es la fuente más usada por cirujanos para ver ejemplos de procedimientos, es comúnmente usada por los estudiantes para aprender anatomía, favorece el aprendizaje independiente, cuenta con una gran aprobación por parte de los estudiantes, puede ayudar a reducir la carga laboral docente y propiciar líneas de investigación. 


\section{Lo aprendido y lo que falta por hacer}

Todo lo estudiado sobre el uso de videos y YouTube en la educación médica parecía prometedor, pero no era suficiente. Requerí investigar sobre la Teoría cognoscitiva del aprendizaje multimedia de Richard Mayer (ver Figura 1), que explica la forma en que procesamos la información que proviene de un medio multimedia (como puede ser un video) y sobre la Teoría de la carga cognitiva de Sweller, que menciona lo fácil que es "sobresaturar" partes de la memoria humana si se usan recursos didácticos mal diseñados (Young, Van Merrienboer, Durning \& Ten, 2014).

Foto 2. Edición del video educativo sobre exploración neurológica básica.

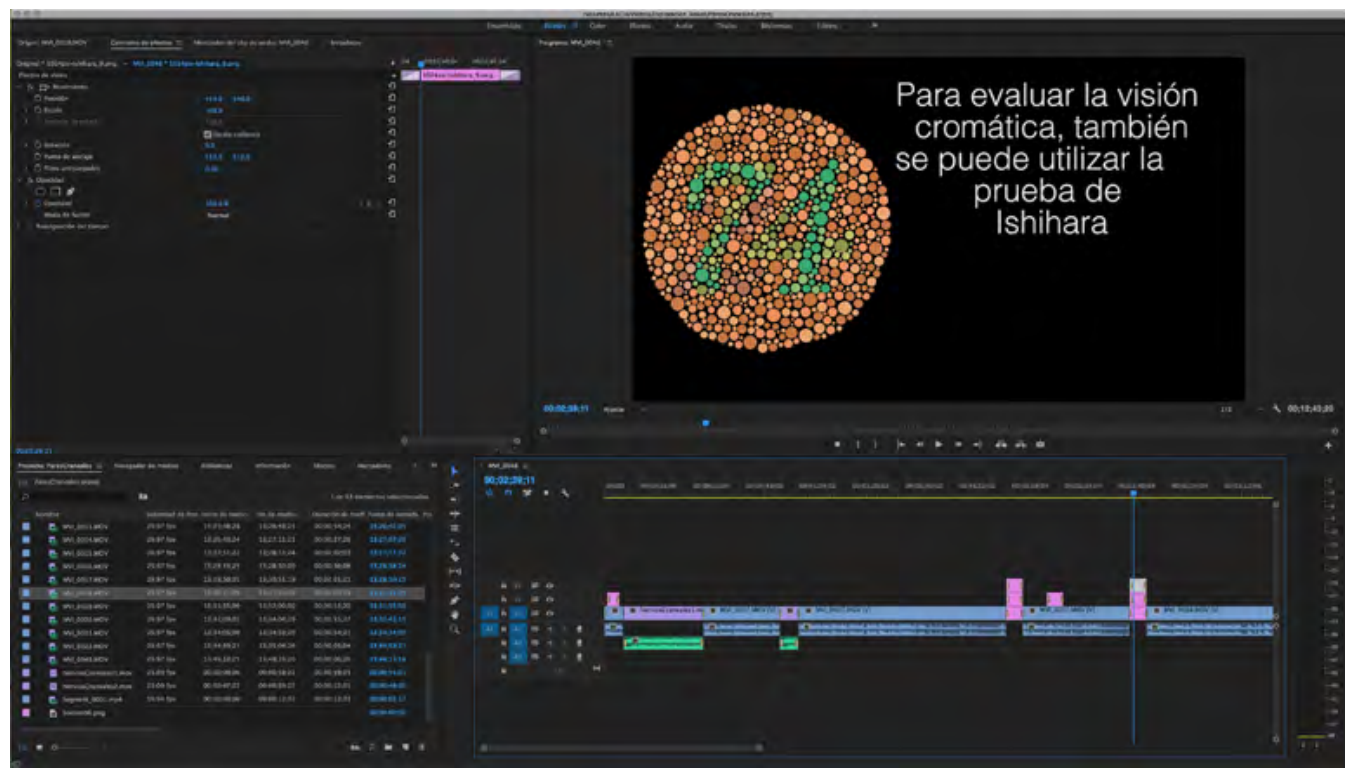

En el momento de redactar este texto llevo la mitad de la maestría cursada; en la cual, he leído mucho para sustentar mi trabajo, además produje un video educativo sobre exploración neurológica básica como parte fundamental de mi proyecto. Han pasado muchas horas de clase, pero sé que todavía falta lo más difícil: aplicar el instrumento, recolectar y analizar datos, obtener y discutir resultados, llegar a conclusiones y, claro, escribir una tesis.

He encontrado obstáculos en el camino, pero la idea de titularme, poder publicar un artículo y desarrollar algo, que genuinamente busque ayudar a los estudiantes, es justo la motivación necesaria para seguir adelante.

\section{Ser un experto en educación}

Después de ser estudiante de pregrado y de posgrado, laborar como docente, haber cursado asignaturas como Docencia en Ciencias de la Salud, Evaluación, Enseñanza de la Clínica o Psicología de la Educación, he comprendido que la Educación 
(sí, con mayúscula) es mucho más que dar clases o tomar clases. La Educación es un universo de posibilidades que hay que explorar. También he reafirmado mis ideas de que ser experto en algo no te vuelve automáticamente un profesor y que en nuestro país hace mucha falta la profesionalización en las áreas educativas.

En un futuro espero poder seguir haciendo videos educativos que ayuden a los estudiantes de medicina y puede que mi canal nunca maneje los números de Yuya, pero la verdad, la etiqueta de youtuber no me llama la atención. Ahora bien, el título de experto en educación, ese sí me parece interesante.

\section{Referencias}

* AMIPCl (2015). Estudio de Hábitos de los Usuarios de Internet en México, 11a Edición. Recuperado de https://www.amipci.org.mx/es/noticiasx/2241-alcanza-internetel-51-de-penetracion-entre-los-usuarios-potenciales-de-mexico-amipci

* Barry, D., Marzuk, F., Chulak-Oglu, K., Bennett, D., Tierney, P. y OKeffe, G. (2016). Anatomy Education for the YouTube Generation. Anat Sci Educ, 9, pp 90-96.

* Donovan, L. (2011). Los millennial en México. Algarabía Pocket, 1, pp 82-96.

- Elam, C., Stratton, T. y Gibson, D. (2007). Welcoming a New Generation to College: The Millennial Students. Journal of College Admission, 1, p 23.

- Jaffar, A. (2012). YouTube: An Emerging Tool in Anatomy Education. Anat Sci Educ, 5, pp 158-164.

* Jaffar, A. (2013). Tips for Using YouTube in Medical Education. Iraqui J Med Sci, 11, pp 102-108.

* Turner, A., Prihoda, T., English, D., Chismark, A. y Jacks, M. (2016). Millennial Dental Hygiene Students' Learning Preferences Compared to Non-Millennial Faculty Members' Teaching Methods: A National Study. Journal of Dental Education, 80 (9), pp 1082-1090.

* La Redacción Proceso. (2012). YouTube en números. Proceso. Recuperado de: http://www.proceso.com.mx/298154/YouTube-en-numeros

* Latapie, I. (2007). Acercamiento al aprendizaje multimedia. Investigación universitaria multidisciplinaria, 6, pp 7-14.

* Rapp, A., Healy, M., Charlton, M., Keith, J., Rosebaum, M. y Kapadia, M. (2016). YouTube is the Most Frequently Used Educational Video Source for Surgical Preparation. Journal of Surgical Education, 1, 1-5.

* Roberts, D., Newman, L. y Schwartzstein, R. (2012). Twelve Tips for Facilitating Millennials' learning. Medical Teacher, 34, pp 274-278.

* Ybarra, R. (2015). Cómo ganan dinero los youtubers y cuáles son los más exitosos. Qore. Recuperado de: http://www.qore.com/articulos/36813/Como-ganandinero-los-youtubers-y-cuales-son-los-mas-exitosos 
* Villamil J. (2016). El nuevo mundo. Proceso, Edición especial, 53, pp 9-10.

- Young, J., Van Merrienboer, J., Durning, S. y Ten, O. (2014). Cognitive Load Theory: Implication for medical education: AMEE Guide No. 86. Medical Teacher, 36, pp 371-384.

- Wilson, M. y Gerber, L. E. (2008). How generational theory can improve teaching: strategies for working with the "millennials". Currents Teach Learn, 1, pp 29-44.

\section{Cómo citar este artículo}

* Massieu Paulin, Alain (2017). De la experiencia digital a la necesidad formativa, Revista Digital Universitaria (RDU), vol. 18, núm. 7, septiembre-octubre. Recuperado de http://dx.doi.org/10.22201/codeic.16076079e.2017.v18n7.a6. 